\title{
VARIACIÓN RADIAL Y AXIAL DEL HINCHAMIENTO, DEL FACTOR ANISOTRÓPICO Y DE LA DENSIDAD, EN EL EUCALYPTUS GRANDIS DE ARGENTINA.
}

\author{
RADIAL AND AXIAL VARIATION OF SWELLING, ANISOTROPY AND \\ DENSITY, IN ARGENTINEAN EUCALYPTUS GRANDIS \\ C.F.Calvo, A.D.Cotrina , A.G.Cuffré , J. C.Piter , P. M.Stefani, E.A.Torrán \\ RESUMEN
}

En este trabajo se presentan los resultados de una investigación orientada a estudiar la variación de los hinchamientos tangencial y radial, así como del coeficiente de anisotropía y la densidad anhidra, en función de la altura y la distancia a la médula de árboles de Eucalyptus grandis cultivados en la Mesopotamia de Argentina. Con este propósito se diseñó y llevó a cabo una investigación empírica con muestras seleccionadas al azar. Los resultados muestran que, para propósitos prácticos, puede asumirse que el hinchamiento y el coeficiente de anisotropía tienen una tendencia decreciente al aumentar la altura, y creciente al aumentar la distancia a la médula. La madera de la periferia exhibe un comportamiento más desfavorable desde el punto de vista de los movimientos dimensionales que la del interior del tronco, si bien presenta mayor densidad y consecuentemente mejor calidad para numerosos empleos.

Palabras clave: Eucalyptus grandis, hinchamiento, coeficiente de anisotropía, densidad.

\begin{abstract}
The present paper reports the results of an investigation regarding the variation in swelling, coefficient of anisotropy and density, as a function of the distance from the pith and the height, in trees of Argentinean Eucalyptus grandis. For this purpose an empirical research project with samples randomly selected was designed and carried out. According to the results and for practical purposes, it may be assumed that swelling and the coefficient of anisotropy decrease with height and increase with the distance from the pith. Wood of the outer part of the tree stem exhibits more problems related to dimensional changes than wood of the inner part of the tree stem, even though it presents higher density and, consequently, higher quality for many purposes.
\end{abstract}

Key words: Eucalyptus grandis, swelling, coefficient of anisotropy, density.

\section{INTRODUCCIÓN}

La anisotropía del hinchamiento y la contracción en las direcciones tangencial y radial ocasiona defectos como deformaciones y grietas, que están relacionados a la ubicación de la pieza en el fuste. Cuanto más grande es la relación expresada como cociente entre el hinchamiento o la contracción entre la dirección tangencial y la radial, mayor es la magnitud del problema. Según Coronel (1994), valores del mencionado cociente, conocido como índice o coeficiente de anisotropía, que se encuentren comprendidos entre 1,2 y 1,5 pueden considerarse excelentes, en tanto que entre 1,6 y 1,9 se destacan como normales y los mayores a 2,0 originan dificultades para determinados usos de la madera.

La presencia de grietas asociadas con problemas de contracción durante el secado exhiben una gran variedad en los eucaliptos, siendo el Eucalyptus grandis la especie que en general presenta menores problemas (Hillis, 1978; INTA, 1995). No obstante, se han reportado deformaciones y grietas que pueden limitar la utilización de esta última especie cultivada en Argentina (Piter et al., 2004).

(1) Director del Grupo de Estudio de Maderas (GEMA), Depto. de Ing. Civil, FRCU, UTN. Ing. Pereira 676, (E3264BTD) C. del Uruguay, E. Ríos, Argentina. (piterj@frcu.utn.edu.ar)

(2) Miembro del Grupo de Estudio de Maderas (GEMA), Depto. de Ing. Civil, FRCU, UTN. Ing. Pereira 676, (E3264BTD) C. del Uruguay, E. Ríos, Argentina.

(3) División Polímeros INTEMA-Facultad de Ingeniería, UNMdP-CONICET. Av. Juan B. Justo 4302, (B7608FDQ), Mar del Plata, Argentina.

Autor para correspondencia: piterj@frcu.utn.edu.ar

Recibido: Octubre 5, 2005. Aceptado: Abril 14, 2006. 
El aumento volumétrico de la madera crece generalmente en forma proporcional con la densidad anhidra (Coronel, 1994; Hoffmeyer, 1995). Para el Eucalyptus grandis de Argentina, que se cultiva mayoritariamente en la Mesopotamia (INTA, 1995), Piter et al. (2004) reportaron valores similares de la densidad que los informados por Pérez del Castillo (2001) para la misma especie cultivada en Uruguay. En el primero de estos casos la edad de los árboles estuvo comprendida entre 16 y 20 años y en el segundo fue de 16,5 años. Estos valores son sensiblemente menores a los adoptados en las normas australianas, si bien en este último caso no se informa la edad de los árboles analizados (Standards Australia, 1986; Standards Australia, 2000). Resultados de investigaciones previas reportan que la elevada relación existente entre las propiedades mecánicas, en particular el módulo de elasticidad, y la densidad, constituye una especial particularidad de esta especie cultivada en Argentina (Piter et al., 2004).

La heterogeneidad que caracteriza a este material hace necesario también estudiar las variaciones dentro de un mismo árbol, en función tanto de la distancia a la médula como de la altura (Coronel, 1994; Hoffmeyer, 1995). El conocimiento del hinchamiento tangencial y radial, a partir de ellos del factor de anisotropía, todos en función de la posición en el árbol, permite optimizar el aserrado con el fin de lograr productos con menores distorsiones y grietas. A su vez, el conocimiento de las variaciones de la densidad permite orientar los cortes a la obtención de piezas de mayor dureza y mejores propiedades mecánicas conforme al destino de las mismas.

El objetivo de este trabajo fue investigar la variación de los valores del hinchamiento tangencial y radial, así como del factor de anisotropía y la densidad anhidra, en función de la distancia a la médula y de la altura en el árbol, en probetas procedentes de plantaciones de Eucalyptus grandis cultivados en la Mesopotamia de Argentina.

\section{MATERIALES Y MÉTODOS}

El material fue obtenido de 4 árboles seleccionados al azar de una plantación de Eucalyptus grandis ubicada en el Departamento de Concordia, Provincia de Entre Ríos. Esta región de cultivo es una de las más importantes del país para la especie (INTA, 1995). La semilla para la plantación fue provista por el INTA de Concordia y los árboles se cortaron con una edad de 17 años. Al momento del corte, los mismos tenían una altura capaz de proveer 6 trozas útiles de 4,0m cada una (ver Figura 1c). El diámetro en el extremo inferior de la troza basal estuvo comprendido entre $40 \mathrm{~cm}$ y $50 \mathrm{~cm}$.

Para el presente trabajo se seleccionaron los extremos inferiores de las trozas, los que fueron estacionados al aire libre en espacio semicubierto hasta alcanzar la humedad de equilibrio higroscópico. Luego de logrado éste, las probetas para la realización de los ensayos de hinchamiento se prepararon con dimensiones $20 \mathrm{~mm}$ x $20 \mathrm{~mm}$ x $50 \mathrm{~mm}$ y orientadas según la dirección del hinchamiento a determinar, a cada una de las cuales se clavaron 4 clavos de bronce de $1,2 \mathrm{~mm}$ de diámetro, de acuerdo con lo estipulado en la norma IRAM 9543 (1966) y conforme al esquema que se presenta en la Figura 1a y 1b. Para calcular la densidad anhidra, las probetas se prepararon con las mismas dimensiones pero sin atender su orientación y omitiendo los clavos antes mencionados. Con el fin de analizar la variación radial de los resultados, las probetas se extrajeron de manera que la distancia entre sus respectivos centros y la médula se corresponda con intervalos de $2,5 \mathrm{~cm}$. Como en todos los casos las mismas se cortaron del extremo inferior de cada troza, las cuales tenían una longitud aproximada de 4,0m, su ubicación respecto de la dirección axial, o sea la altura en el árbol, se definió para intervalos de 4,0m a partir del punto inferior de la troza basal, como se indica en la Figura 1c. 


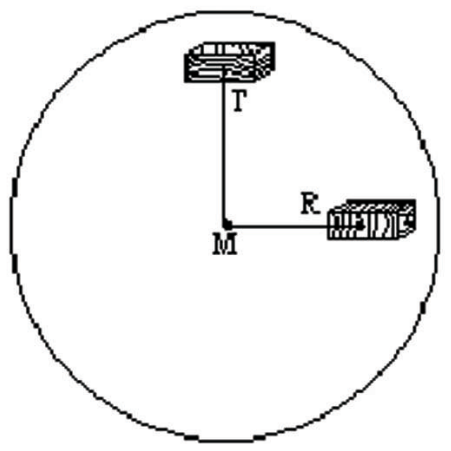

a)

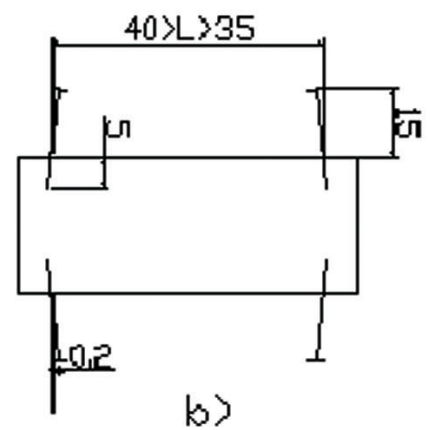

b)

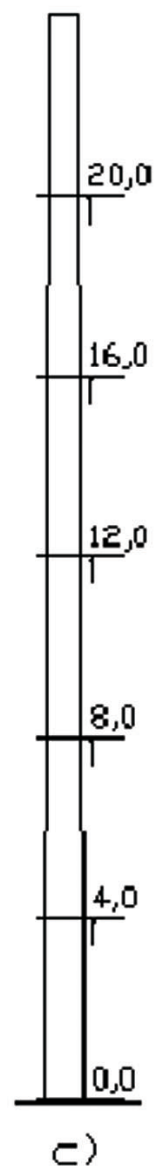

c)

Fig. 1: Extracción y preparación de las probetas

a) Extracción de las probetas para medir el hinchamiento. T: tangencial; R: radial. La distancia desde la médula (M) se midió hasta su punto medio en intervalos de $2,5 \mathrm{~cm}$

b) Esquema de ubicación de los clavos (dimensiones en mm)

c) Medición de la altura de las probetas en el árbol, en intervalos de 4,0m a partir del punto inferior de la troza basal

La cantidad de probetas preparadas y utilizadas para determinar el hinchamiento en las direcciones tangencial y radial, así como para calcular la densidad anhidra, fue de 193, 170 y 78, respectivamente. La cantidad de determinaciones del coeficiente de anisotropía fue de 40. En la Tabla 1 se muestra una discriminación por posición respecto de la médula y de la altura en el árbol, así como por tipo de determinación realizada. 
Tabla 1: Cantidad de probetas y determinaciones del coeficiente de anisotropía

\begin{tabular}{|c|c|c|c|c|c|}
\hline $\begin{array}{l}\text { Altura } \\
(\mathrm{m})\end{array}$ & $\begin{array}{c}\text { Distancia a la } \\
\text { médula }(\mathrm{cm})\end{array}$ & $\mathrm{T}$ & $\mathrm{R}$ & $\mathrm{CA}$ & D \\
\hline \multirow{5}{*}{0,0} & 2.5 & 15 & 10 & 3 & 0 \\
\hline & 5 & 12 & 8 & 2 & 6 \\
\hline & 7.5 & 11 & 8 & 2 & 6 \\
\hline & 10 & 16 & 12 & 3 & 5 \\
\hline & 12.5 & 13 & 4 & 1 & 4 \\
\hline \multirow{5}{*}{4,0} & 2.5 & 7 & 6 & 2 & 0 \\
\hline & 5 & 7 & 10 & 2 & 5 \\
\hline & 7.5 & 6 & 12 & 2 & 6 \\
\hline & 10 & 7 & 0 & 0 & 6 \\
\hline & 12.5 & 0 & 0 & 0 & 2 \\
\hline \multirow{5}{*}{8,0} & 2.5 & 12 & 14 & 3 & 2 \\
\hline & 5 & 15 & 14 & 4 & 4 \\
\hline & 7.5 & 11 & 10 & 3 & 4 \\
\hline & 10 & 7 & 0 & 0 & 4 \\
\hline & 12.5 & 0 & 0 & 0 & 0 \\
\hline \multirow{5}{*}{12,0} & 2.5 & 8 & 15 & 3 & 0 \\
\hline & 5 & 11 & 15 & 3 & 4 \\
\hline & 7.5 & 12 & 8 & 1 & 4 \\
\hline & 10 & 0 & 4 & 0 & 0 \\
\hline & 12.5 & 0 & 0 & 0 & 0 \\
\hline \multirow{5}{*}{16,0} & 2.5 & $\frac{6}{6}$ & 6 & 2 & 0 \\
\hline & 5 & 7 & 6 & 2 & 6 \\
\hline & 7.5 & 0 & 0 & 0 & 6 \\
\hline & 10 & 0 & 0 & 0 & 0 \\
\hline & 12.5 & 0 & 0 & 0 & 0 \\
\hline \multirow{5}{*}{20,0} & 2.5 & 4 & 4 & 1 & 0 \\
\hline & 5 & 4 & 4 & 1 & 2 \\
\hline & 7.5 & 2 & 0 & 0 & 2 \\
\hline & 10 & 0 & 0 & 0 & 0 \\
\hline & 12.5 & 0 & 0 & 0 & 0 \\
\hline
\end{tabular}

T: tangencial; R: radial; CA coeficiente de anisotropía; D: densidad anhidra

Las probetas destinadas al cálculo del hinchamiento se almacenaron durante un mínimo de 7 días en clima normalizado, con temperatura de $20(+/-2)^{\circ} \mathrm{C}$ y humedad relativa ambiente de $65(+/-5) \%$, logrando un contenido de humedad cercano al $12 \%$. Posteriormente, se colocaron en estufa a temperatura ambiente, la que luego se fue elevando paulatinamente hasta alcanzar $100(+/-3)^{\circ} \mathrm{C}$ en un tiempo no menor a 24 horas. Cuando se logró peso constante, en estado anhidro, se midió la distancia entre los clavos de cada cara, calculándose su promedio, que se denominó L'. El procedimiento empleado es el adoptado por la norma IRAM 9543 (1966) para madera estacionada al aire, y en el presente trabajo no se manifestó la presencia de colapso, por lo cual no fue necesario emplear tratamientos de reacondicionamiento. Posteriormente se sumergieron las probetas en agua, con excepción de una superficie transversal, permitiendo la penetración del agua a través de la cara inferior, y el desplazamiento del aire hacia la 
superior. Una vez húmeda esta última, se sumergieron por completo, permaneciendo de esta forma un mínimo de 7 días. Lograda la saturación, se retiraron del agua y se secaron con un trapo húmedo, para posteriormente medir la distancia entre clavos en cada cara, designando su promedio con L". Para la determinación de las dimensiones se utilizó un calibre digital capaz de registrar $0,01 \mathrm{~mm}$ y para pesar las probetas se empleó una balanza electrónica capaz de registrar 0,01g. El hinchamiento lineal máximo de cada cuerpo de prueba, expresado como porcentaje, fue determinado utilizando la siguiente ecuación:

$$
\alpha_{i}=\frac{\left(L^{\prime \prime}{ }_{i}-L_{i}^{\prime}\right)}{L_{i}^{\prime}} \times 100
$$

donde:

$\alpha_{i}$ : hinchamiento lineal máximo para la dirección correspondiente (tangencial o radial) expresado como porcentaje

$L{ }_{i}$ : distancia promedio entre clavos, con la probeta en estado saturado, para la dirección correspondiente

$L_{i}^{\prime}:$ distancia promedio entre clavos, con la probeta en estado anhidro, para la dirección correspondiente

Teniendo en cuenta que las determinaciones del hinchamiento se efectuaron siempre según la dirección de mayor longitud (50mm) de las probetas, conforme lo estipulado en la norma IRAM 9543 (1966), el coeficiente de anisotropía se determinó para cada posición, tanto en altura como respecto de la médula, en un mismo árbol. Su cálculo se efectuó dividiendo los promedios del hinchamiento máximo correspondientes a las direcciones tangencial y radial para cada posición dentro de cada árbol, siempre que existiesen valores de ambas variables.

La densidad anhidra se calculó como el cociente entre la masa y el volumen en las probetas correspondientes, ambos medidos en estado anhidro, conforme al procedimiento indicado en la norma ISO 3131 (1975), empleando los instrumentos de medición mencionados anteriormente.

Con los datos obtenidos se realizó una evaluación estadística orientada a obtener el valor medio como medida de tendencia central y el coeficiente de variación (COV) como medida de dispersión.

\section{RESULTADOS Y DISCUSIÓN}

La Tabla 2 exhibe, en función de la ubicación de las probetas en el árbol, los principales resultados para el hinchamiento lineal máximo, determinado entre el estado anhidro y el de saturación de las fibras, así como para el coeficiente de anisotropía y para la densidad anhidra.

Se han publicado pocos datos referidos al hinchamiento de esta especie cultivada en Argentina. Calvo et al. (2004) reportan valores medios para el hinchamiento tangencial máximo que oscilan entre $9,8 \%$ y $11,5 \%$, con coeficientes de variación comprendidos entre $13,5 \%$ y $21,9 \%$, para 5 plantaciones de Eucalyptus grandis de la Mesopotamia de Argentina. Los mismos autores publican valores medios para el hinchamiento radial que oscilan entre $4,5 \%$ y $7,1 \%$, con coeficientes de variación comprendidos entre $21,6 \%$ y 33,2\%. Para las especies Eucalyptus camaldulensis y Eucalyptus tereticornis, ambas cultivadas en el Parque Chaqueño de Argentina, Coronel (1994) reporta datos referidos al hinchamiento lineal máximo. Para la primera especie, informa un valor medio del hinchamiento tangencial igual a 9,9\% y del radial igual a 4,9\%, con coeficientes de variación de $17,5 \%$ y 36,6\%, respectivamente. Para la segunda, los correspondientes valores medios son $11,9 \%$ y $6,7 \%$, con coeficientes de variación de 34,5\% y $30,4 \%$, respectivamente. 
Tabla 2: Resultados discriminados por altura en el árbol y distancia a la médula

\begin{tabular}{|c|c|c|c|c|c|}
\hline $\begin{array}{l}\text { Altura } \\
(\mathrm{m})\end{array}$ & $\begin{array}{l}\text { Distancia a la } \\
\text { médula }(\mathrm{cm})\end{array}$ & $\mathrm{T}$ & $\mathrm{R}$ & $\mathrm{CA}$ & $\mathrm{D}$ \\
\hline \multirow{5}{*}{0,0} & 2.5 & $10,7(13)$ & $6,8(27)$ & $1,53(32)$ & \\
\hline & 5 & $12,0(24)$ & $7,6(35)$ & $1,44(27)$ & $541(16)$ \\
\hline & 7.5 & $12,2(17)$ & $5,8(27)$ & $2,03(12)$ & $566(11)$ \\
\hline & 10 & $13,8(17)$ & $7,6(24)$ & $1,82(8)$ & $605(13)$ \\
\hline & 12.5 & $13,0(19)$ & $6,3(8)$ & 1,52 & $634(6)$ \\
\hline \multirow{5}{*}{4,0} & 2.5 & $8,4(13)$ & $5,8(17)$ & $1,45(12)$ & \\
\hline & 5 & $9,3(5)$ & $4,9(19)$ & $1,93(5)$ & $433(6)$ \\
\hline & 7.5 & $10,6(3)$ & $7,0(18)$ & $1,52(2)$ & $507(17)$ \\
\hline & 10 & $13,1(7)$ & & & $585(8)$ \\
\hline & 12.5 & & & & $632(2)$ \\
\hline \multirow{5}{*}{8,0} & 2.5 & $7,2(6)$ & $5,3(17)$ & $1,42(15)$ & $488(20)$ \\
\hline & 5 & $8,8(11)$ & $5,8(20)$ & $1,53(19)$ & $449(3)$ \\
\hline & 7.5 & $10,4(6)$ & $8,0(13)$ & $1,32(16)$ & $508(8)$ \\
\hline & 10 & $10,9(7)$ & & & $594(6)$ \\
\hline & 12.5 & & & & \\
\hline \multirow{5}{*}{12,0} & 2.5 & $7,0(10)$ & $5,6(22)$ & $1,34(14)$ & \\
\hline & 5 & $8,3(11)$ & $6,6(15)$ & $1,23(5)$ & $542(10)$ \\
\hline & 7.5 & $9,8(9)$ & $6,2(17)$ & 1,27 & $559(20)$ \\
\hline & 10 & & $7,3(5)$ & & \\
\hline & 12.5 & & & & \\
\hline \multirow{5}{*}{16,0} & 2.5 & $7,6(6)$ & $5,8(6)$ & $1,33(0)$ & \\
\hline & 5 & $8,5(6)$ & $6,7(11)$ & $1,24(7)$ & $560(10)$ \\
\hline & 7.5 & & & & $625(14)$ \\
\hline & 10 & & & & \\
\hline & 12.5 & & & & \\
\hline \multirow{4}{*}{20,0} & 2.5 & $7,6(9)$ & $5,6(7)$ & 1,37 & \\
\hline & 5 & $7,7(4)$ & $5,8(10)$ & 1,33 & $543(9)$ \\
\hline & 7.5 & $8,2(4)$ & & & $618(10)$ \\
\hline & $\frac{10}{12.5}$ & & & & \\
\hline
\end{tabular}

Se expresan los valores medios para cada posición. T: hinchamiento tangencial (\%); R: hinchamiento radial (\%); CA coeficiente de anisotropía; D: densidad anhidra $\left(\mathrm{kg} / \mathrm{m}^{3}\right)$; entre paréntesis se indica el coeficiente de variación $(\%)$

El rango de variación de los resultados encontrados en este trabajo para el coeficiente de anisotropía indica un comportamiento normal para esta propiedad, de acuerdo con el criterio adoptado por Coronel (1994). En la misma línea, Hillis (1978), afirma que el Eucalyptus grandis de Nueva Zelanda y Sudáfrica puede ser secado satisfactoriamente, refiriéndose a su comportamiento anisotrópico. Para el Eucalyptus grandis cultivado en Brasil, ABPM (2001) publica un coeficiente de anisotropía de 2,1.

Los valores medios encontrados para la densidad anhidra son similares a los reportados por Pérez del Castillo (2001) para madera de Eucalyptus grandis obtenida de árboles de 16,5 años cultivados en el norte de Uruguay y a los informados por Piter et al. (2004) para árboles de la misma especie cultivada en Argentina, con edad comprendida entre 16 y 20 años. A su vez, son sensiblemente menores a los publicados en Australia para la misma especie, aunque sin indicar la edad de los árboles analizados (Standards Australia, 1986; Standards Australia, 2000).

Un análisis detallado de los valores presentados en la Tabla 2 muestra que en general el hinchamiento tangencial y la densidad crecen con la distancia a la médula para una misma altura dentro del árbol. A su vez, las variaciones experimentadas en función de la altura para una misma distancia desde la médula son de menor importancia que las anteriores. Este comportamiento es coherente con resultados de la densidad publicados por Hillis (1978) para la misma especie de distintas procedencias. El mismo análisis detallado evidencia que los valores medios del hinchamiento radial no muestran una tendencia tan clara 
como la exhibida por las dos variables mencionadas anteriormente.

Con el fin de determinar la variación de las propiedades en forma general, persiguiendo propósitos prácticos, se analizó la evolución de las mismas en función de la altura, considerando en forma conjunta todas las posiciones respecto de la médula, y viceversa. La variación global de las propiedades en función de la altura puede ser apreciada en las figuras 2 y 3. Los valores de estas figuras no coinciden con los de la Tabla 2 porque en las figuras se presentan para cada altura, en forma conjunta, los resultados de todas las distancias respecto de la médula.

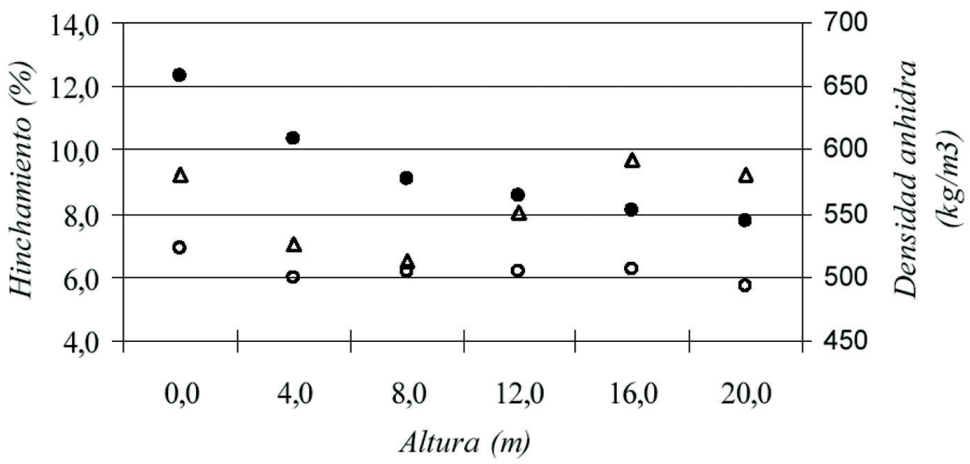

Fig. 2: Valores del hinchamiento y la densidad anhidra en función de la altura

Se expresan los valores medios de los resultados correspondientes a cada altura, sin discriminar la distancia desde la médula. $\bullet$ : hinchamiento tangencial; o: hinchamiento radial; $\Delta$ : densidad anhidra.

Los valores medios del hinchamiento tangencial muestran un descenso al aumentar la altura. La disminución es más pronunciada entre el nivel inferior de la troza basal, o sea $0,0 \mathrm{~m}(12,4 \%), \operatorname{los} 4,0 \mathrm{~m}$ $(10,3 \%)$, y los $8,0 \mathrm{~m}(9,1 \%)$, para luego atenuarse hasta $8,6 \%, 8,1 \%$ y $7,8 \%$ en los $12,0 \mathrm{~m}, 16,0 \mathrm{~m}$ y $20,0 \mathrm{~m}$, respectivamente. El hinchamiento radial disminuye desde $6,9 \%$ en la base a $6,0 \%$ a los $4,0 \mathrm{~m}$ de altura, para luego aumentar levemente y estabilizarse en $6,2 \%$ a los $8,0 \mathrm{~m}, 12,0 \mathrm{~m}, \mathrm{y} 16,0 \mathrm{~m}$, y finalmente disminuir a 5,7\% a los 20,0m. Las variaciones encontradas en función de la altura para el hinchamiento son congruentes con las reportadas por Calvo et al. (2004) para la misma especie. La densidad anhidra muestra un comportamiento similar al del hinchamiento radial, con valores de $581 \mathrm{~kg} / \mathrm{m}^{3}, 525 \mathrm{~kg} / \mathrm{m}^{3}$, $513 \mathrm{~kg} / \mathrm{m}^{3}, 550 \mathrm{~kg} / \mathrm{m}^{3}, 592 \mathrm{~kg} / \mathrm{m}^{3}$ y $581 \mathrm{~kg} / \mathrm{m}^{3}$. En este caso la relación entre las variaciones dimensionales y el valor de la densidad (Coronel, 1994; Hoffmeyer, 1995) se verifica más para el hinchamiento radial que para el tangencial.

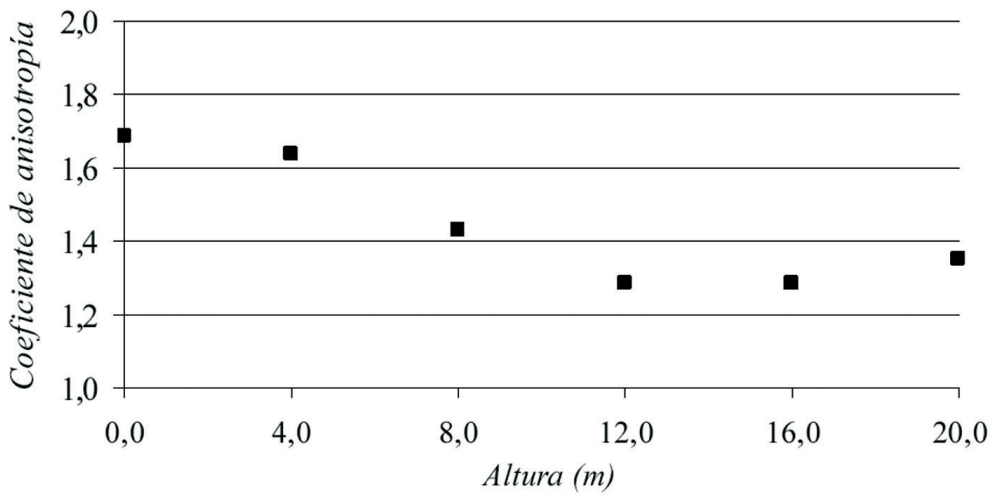

Fig. 3: Valores del coeficiente de anisotropía en función de la altura

Se expresan los valores medios de los resultados correspondientes a cada altura, sin discriminar la distancia desde la médula. 
Los valores medios de las determinaciones del coeficiente de anisotropía (ver Figura 3) presentan un leve descenso entre el nivel inferior $(1,68)$ y los 4,0m $(1,63)$, para luego mostrar disminuciones más acentuadas a los $8,0 \mathrm{~m}(1,43)$ y $12,0 \mathrm{~m}(1,28)$, estabilizarse hasta los $16,0 \mathrm{~m}(1,29)$ y finalmente crecer levemente en los 20,0m $(1,35)$. Estos resultados confirman un mejor comportamiento de la madera para las trozas superiores que para las inferiores desde el punto de vista anisotrópico.

La variación global de las propiedades investigadas en función de la distancia a la médula puede ser apreciada en las figuras 4 y 5. Los valores de estas figuras no coinciden con los de la Tabla 2 porque en las figuras se presentan para cada distancia desde la médula, en forma conjunta, los resultados de todas las alturas consideradas. Los valores medios del hinchamiento tangencial muestran un incremento para las 5 posiciones analizadas al aumentar la distancia desde la médula. Los valores son 8,4\% para la distancia de $2,5 \mathrm{~cm}, 9,3 \%$ para $5,0 \mathrm{~cm}, 10,7 \%$ para $7,5 \mathrm{~cm} 12,9 \%$ para $10,0 \mathrm{~cm}, \mathrm{y}$, finalmente, $13,0 \%$ a los $12,5 \mathrm{~cm}$. Los valores medios del hinchamiento radial crecen desde 5,8\% (2,5cm), a 6,2\% (5cm), a $6,8 \%$ $(7,5 \mathrm{~cm})$ y hasta $7,5 \%(10,0 \mathrm{~cm})$ para luego disminuir a $6,6 \%(12,5 \mathrm{~cm})$. Estos resultados son también coherentes con los publicados por Calvo et al. (2004) en la investigación previa con la misma especie ya mencionada anteriormente. La densidad aumenta desde el centro hacia la periferia en este caso para todas las posiciones analizadas, presentando valores de $488 \mathrm{~kg} / \mathrm{m}^{3}, 512 \mathrm{~kg} / \mathrm{m}^{3}, 560 \mathrm{~kg} / \mathrm{m}^{3}, 594 \mathrm{~kg} / \mathrm{m}^{3}$ y $633 \mathrm{~kg} / \mathrm{m}^{3}$. En línea con las afirmaciones de Coronel (1994) y Hoffmeyer (1995), las variaciones dimensionales aumentan al aumentar la densidad, y en este caso lo hacen en función de la distancia a la médula.

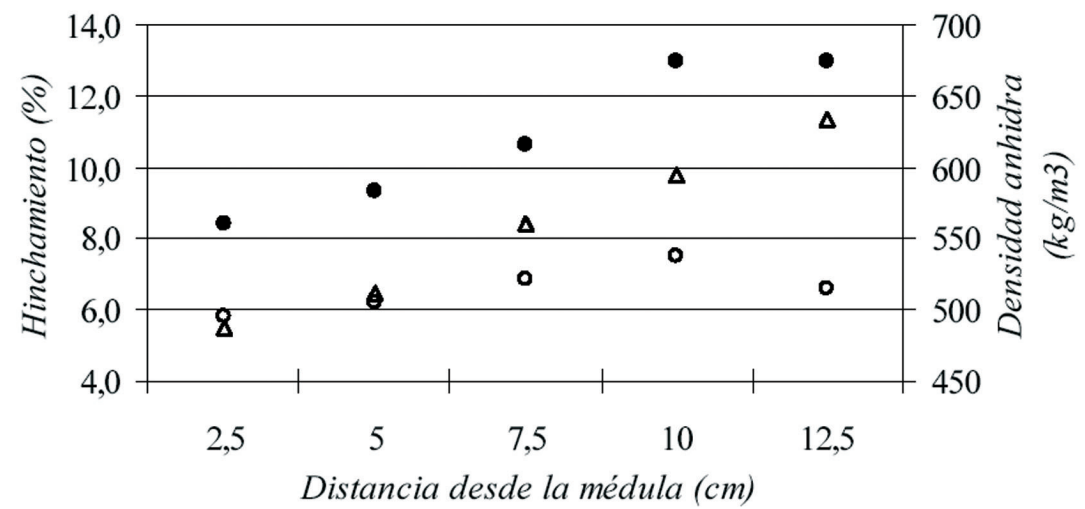

Fig. 4: Valores del hinchamiento y la densidad anhidra en función de la distancia desde la médula

Se expresan los valores medios de los resultados correspondientes a cada distancia desde la médula, sin discriminar la altura. $\bullet$ : hinchamiento tangencial; o: hinchamiento radial; $\Delta$ : densidad anhidra.

El coeficiente de anisotropía (ver Figura 5) aumenta con la distancia desde la médula desde 1,42 para $2,5 \mathrm{~cm}$, a 1,45 para $5,0 \mathrm{~cm}$, hasta 1,54 para $7,5 \mathrm{~cm}$, acentuando luego el incremento hasta 1,82 a $10,0 \mathrm{~cm}$ y disminuir finalmente a $1,52 \mathrm{en} 12,5 \mathrm{~cm}$. Los resultados confirman un mejor comportamiento de la madera más cercana al centro desde el punto de vista anisotrópico, aunque presente menor resistencia y rigidez que la de la zona externa, como ha sido informado en publicaciones previas (Piter et al., 2004). 


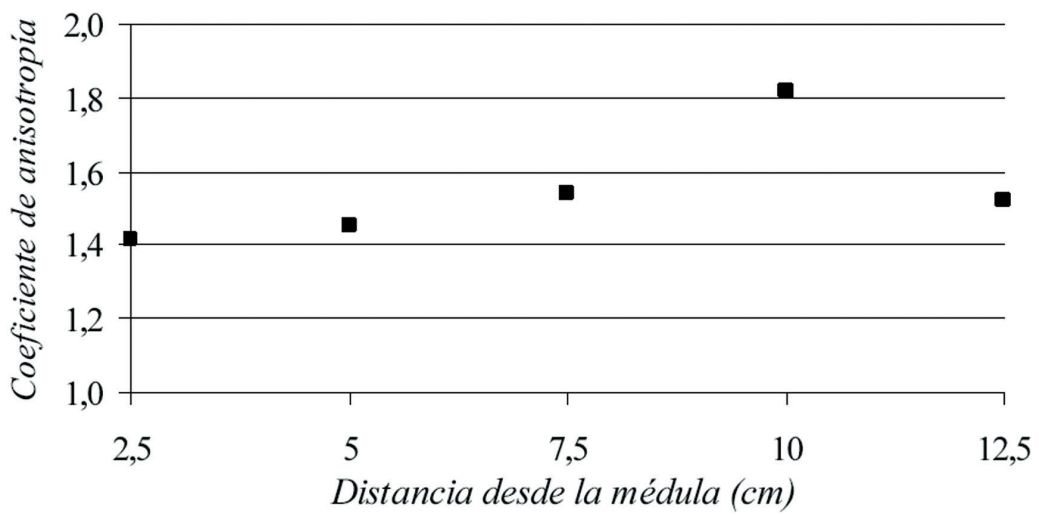

Fig. 5: Valores del coeficiente de anisotropía en función de la distancia desde la médula.

Se expresan los valores medios de los resultados correspondientes a cada distancia desde la médula, sin discriminar la altura.

\section{CONCLUSIONES}

Fue posible estudiar el comportamiento del hinchamiento, el coeficiente de anisotropía y la densidad anhidra en función de la altura en el árbol y la distancia a la médula en muestras de Eucalyptus grandis cultivado en la Mesopotamia de Argentina. Los hinchamientos tangencial y radial máximos presentan magnitudes coherentes con resultados publicados por otros investigadores para la especie. El coeficiente de anisotropía alcanza niveles normales, confirmando que la especie puede ser secada y procesada sin riesgos excesivos de que se produzcan defectos asociados a esta propiedad. La densidad presenta valores coherentes con otros publicados para la especie cultivada en Argentina y Uruguay, pero sensiblemente menores a los reportados para la misma en Australia. Tanto la magnitud de las variaciones dimensionales como el nivel del coeficiente de anisotropía muestran, en general y para propósitos prácticos, una tendencia decreciente al aumentar la altura, y una tendencia creciente al aumentar la distancia a la médula. Si bien la madera de la periferia presenta mayor densidad y más elevadas propiedades mecánicas, desde el punto de vista de su estabilidad dimensional los resultados indican un comportamiento más problemático que la que procede de la zona central.

\section{BIBLIOGRAFÍA}

Associaçâo Brasileira de Produtores de Madeira - ABPM. 2001. A retractibilidade na madeira, Eucalipto, Revista da Madeira, setiembre 2001, p. 68-70, Curitiba, PR, Brasil.

Calvo, C.F.; Cotrina, A.D.; Cuffre, A.; Ott, M.; Piter, J.C.; Stefani, P.M.; Torrán, E.A.; Vivian, A.C.. 2004. Propiedades de hinchamiento y contracción en la madera de Eucalyptus grandis de Argentina. Cuiabá, Brasil, IX EBRAMEM.

Coronel, E. O.. 1994. Fundamentos de las propiedades físicas y mecánicas de las maderas, $1^{\circ}$ parte, fundamentos de las propiedades físicas de las maderas. Instituto de Tecnología de la Madera, Facultad de Ciencias Forestales, Universidad Nacional de Santiago del Estero, 1ed. Santiago del Estero.

Hillis, W. E.. 1978. Wood quality and utilization. In Eucalypts for Wood Production, eds. W. E. Hillis and A. G. Brown, pp. 258-289. CSIRO, Melbourne.

Hoffmeyer, P.. 1995. Wood as a building material. In Timber Engineering STEP 1, pp. A4/1-A4/21. Centrum Hout, The Netherlands.

Instituto Argentino de Normalización - IRAM. 1966. IRAM 9543 - Método de determinación de las contracciones totales, axil, radial y tangencial y el punto de saturación de las fibras. IRAM, Buenos Aires. 
Instituto Nacional de Tecnología Agropecuaria - INTA. 1995. Manual para Productores de Eucaliptos de la Mesopotamia Argentina. Grupo Forestal, EEA INTA Concordia, Argentina.

International Organization for Standardization. 1975. ISO 3131-1975 (E), Wood - Determination of density for physical and mechanical tests.

Pérez del Castillo, A.. 2001. Propiedades Mecánicas y Calidad de Madera de Eucalyptus Grandis del Norte de Uruguay. Informe de Investigación Nº, LATU, Montevideo.

Piter, J. C.; Zerbino, R. L.; Blaß, H. J. 2004. Visual strength grading of Argentinean Eucalyptus grandis. Strength, stiffness and density profiles and corresponding limits for the main grading parameters. Holz als Roh und Werkstoff 62:1-8.

Standards Australia. 1986. AS 2878-1986, Timber - Classification into strength groups. Standards Australia, North Sydney, N.S.W.

Standards Australia. 2000. AS 2082/2000, Timber - Hardwood - Visually stress-graded for structural purposes. Standards Australia, Australia. 